\title{
Delivering Multimedia in Autonomic Networking Environments
}

\author{
Vassilios Kaldanis ${ }^{1}$, Ranganai Chaparadza ${ }^{2}$, Giannis Katsaros ${ }^{1}$, \\ and George Karantonis ${ }^{1}$ \\ ${ }^{1}$ VELTI S.A. Mobile Marketing and Advertising, \\ 42 Kifissias Ave, 15125 Maroussi, Athens, Greece, \\ \{vkaldanis, gkatsaros, gkar\} @velti.com \\ ${ }^{2}$ Fraunhofer FOKUS, Institute for Open Communcation Systems, \\ 31 Kaiserin-Augusta-Allee, D-10589, Berlin, Germany \\ Ranganai.Chaparadza@fokus. fraunhofer.de
}

\begin{abstract}
This paper aims to investigate the most important aspects, features and requirements around the delivery of Multimedia services in cross-layer architectures that can be exploited for the development of autonomic service management middleware on the top of autonomic networking environments. Fundamental autonomic features like self-management, self-healing and self-adaptation are closely (re) considered and analyzed through the prism of network agnostic media adaptation and other application-layer management mechanisms with the aim to indentify the expected impact of autonomics in end user service deployment experience. The work describes on a high level the specific features and requirements of a proposed autonomic service management framework for the support of steaming media applications in fixed and mobile autonomic accessed networking environments. The work is part of the EFIPSANS project one of the largest research project in IPv6 enabled autonomic networks and Extensions to IPv6 $(I P v 6++)$ towards the Self-Managing Future Internet.
\end{abstract}

Keywords: Autonomic Networking, Service Management, Self-Management, Self-Adaptation, Med.

\section{Introduction}

Autonomicity realized through control-loop structures operating within network nodes/devices and the network as a whole, is an enabler for advanced and enriched self-manageability of network devices and networks. We define an autonomic behaviour as follows: It is a behaviour or action that may consist of a set of sub-behaviours or sub-actions, triggered by what is called an Decision-Making Element (DME) or Decision Element (DE) in an attempt to achieve the goal defined by how the DME manages a Managed Entity (ies) or ME(s) under its control. The autonomic behaviour is considered as a behaviour of the DE or DME (combined DE/DME), triggered as a result of reception of information from its information suppliers such as its associated Managed Entity (ies), in an attempt to regulate or reconfigure the behaviour of the Managed Entity (ies). An example of an autonomic behaviour is: self-description and 
self-advertisement, self-healing, self-configuration, all triggered by an DecisionMaking Element. Therefore, it is important to note that an autonomic behaviour binds to a Decision-Making Element, and possibly (though not necessarily) to information supply parts of the control-loop implemented by the Decision-Making Element together with the Managed Entity (ies) under the control of the Decision-Making Element. A Decision-Making Element can be introduced as standalone entity in a node or as part of a functional entity such as a protocol or application.

This paper attempts through a high-level approach, to identify the different aspects and perspectives of multimedia delivery, both in fixed/wireless and mobile access environments that can be exploited in the design of an autonomic self-managed and self-adapted system. To this end it proposes an autonomic mechanism for the efficient self-management of multimedia services and applications taking advantage at the highest possible level the existing underlying autonomic networking environment. Important issues across application to transport layer like media and network adaptation, resource utilization, and congestion control are under focus in cross-layered architectures as appear today. Our purpose is to provide an innovative framework that will effectively and autonomously manage well known protocols like SIP/SDP, RTP/RTCP, and RTSP, while at the same time being tightly coupled with an also autonomous networking environment.

End users deploying pervasive services on the top an IPv6/IPv6++ autonomic and heterogeneous network expect to meet certain levels of QoS and QoE or performance conditions. Our approach aims at dealing with all required underlying network management and control issues and complexities in order to deliver a reliable and efficiently self-manageable transport layer that will transparently support a wide range of end user applications. Isolation of end users from any form of network management is the most important requirement that will allow the deployment of pervasive multimedia services in the form of context aware applications.

The field of autonomics is expected to highly influence the effective wedding of the two major fields of service and network management together into a self tuned and self-managing network. The proposed mechanisms aim at a first stage to address all required autonomic network management issues around QoS and resource availability as well as the lower layer aspects like connectivity, routing etc as well. The framework also provides an advanced performance monitoring mechanism capable to measure a number of autonomic oriented metrics and parameters both in network and service/application part. The running application on the top of the autonomic node highly relies on the collected performance data to feedback the corresponding autonomic mechanisms in order to:

- Self-adapt to the changing network conditions

- Self-manage the current application session (media)

- Self-heal from severe network conditions (e.g. congestion)

Finally the study provides a unified methodology for the evaluation and assessment of the impact of autonomic behaviors on application performance, providing the mechanism for collecting the end-to-end performance data of applications and the transfer of these performance metrics to the autonomic node in order to adapt the application. The GANA based autonomic service management framework along with the former performance estimation strategy consist a complete framework for the evaluation of autonomic communications systems. 
For comparison purposes in [7] a similar work is also presented as an early attempt to address automated management of services. There the proposed architecture ensures the delivery of services according to specific service level agreements (SLAs) between customers and service providers. Our approach takes a rather more realistic view maintaining the distinction between the service and the network layers capturing the required interfaces and interactions among the proposed autonomic entities and the individual underling protocols as managed entities.

The work presented in this paper is structured as follows: in chapter 2 the particular aspects and parameters of the multimedia delivery mechanisms in cross-layer architectures are presented focusing on the media management and adaptation approaches today. In chapter 3 a brief analysis of the Generic Autonomic Network Architecture (GANA) is presented with special attention on the higher layer components directly involved with the service management processes. In chapter 4 the desired autonomic features are interpreted in the language of multimedia application and service management as presented in chapter 2. Then a detailed analysis of the GANA compatible Service Management framework is provided along with requirements description.

\section{Aspects of Multimedia Delivery in Cross-Layered Environments}

Delivering multimedia over the Internet is a quite challenging task that requires specific requirements to be met in many different parts of the network, the most important being the transmission, the transport and the network access. Successful delivery typically imposes strict QoS requirements on bandwidth, delay and delay jitter and only best-effort service can be supported assuming constantly varying network conditions. Such variation becomes more unpredictable and rapid in the case of wireless and mobile transmission where mobility further affects network conditions by moving between servicing areas with limited resource availability and connectivity-making delivery more difficult. In order to improve perceived multimedia quality and guarantee delivery by end users over wired and wireless/mobile Internet QoS provision is performed across different layers ranging from application, transport to data link layer following the so-called cross-layered approach. Different layers impacts differently the delivered multimedia quality and therefore they impose different approaches for improving delivery depending on the case.

In [2] a quite enlightening diagram of a typical cross-layer architecture and involved layers in different components for multimedia delivery has been borrowed from [2] and is provided in the following figure (fig 1).

The three key components in a wireless Internet architecture for multimedia delivery are: the multimedia server, the Base Station (BS) and the mobile hosts. In the multimedia server component the application layer is responsible for the session management using the key functionalities: network aware media adaptation and the adaptive error control.

The network aware media adaptation functionality determines the media codec type that is used depending the detected network conditions. It has the ability to dynamically change the coding rate and other coding parameters adapting in this way to the varying network conditions (bandwidth, loss, delay etc). Furthermore scalable coding techniques are also introduced to realized this type of media adaptation the most important of 
which is layered coding (e.g. H.263 and MPEG-4). The full details of media coding functionality although here gets out of scope however it is of major importance that the application server gets constant feedback about the current network conditions on the reception end and based on that it selects the appropriate media coding scheme to transport the multimedia content to the end user dynamically during the session.

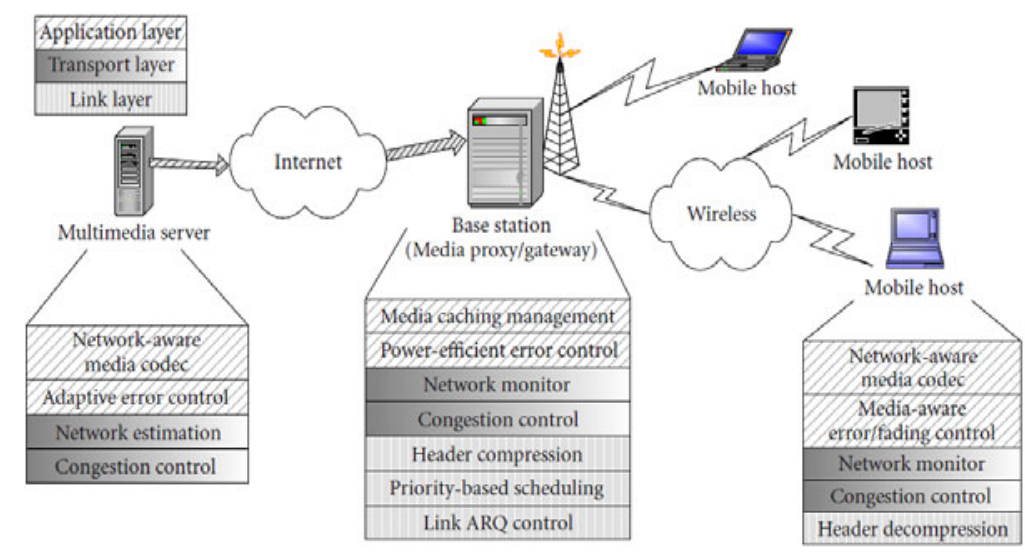

Fig. 1. Cross-layer architecture for multimedia delivery over wireless Internet (source [2])

In the case of adaptive error control it is mainly used to shield delay sensitive applications like voice in the case of problematic wireless transmission where efficient channel coding techniques are required (e.g. ARQ and FEC) to protect data integrity.

To complete the puzzle, in the multimedia server component the transport layer or multimedia transmission control layer is responsible to efficiently deliver the service to the end user at the best possible quality. To accomplish this role it is important to estimate the status of all underlying networks so that multimedia can adapt accordingly. This is achieved through the deployment of specific streaming control transport protocols for the monitoring and control of the media streaming process like the IETF real-time transport control protocol (RTP/RTCP), real-time streaming protocol (RTSP), session initiation protocol (SIP), session description protocol (SDP), and streaming control transport protocol (SCTP). The degree at which these protocols are able to achieve a desirable media streaming quality relies on the accuracy of the estimation of the network conditions.

Estimation of network conditions in practice is performed through detection of current available bandwidth and application of efficient congestion control. A proper congestion control scheme should maximize the bandwidth utilization and at the same time avoid overusing network resource which may cause network collapse. Using a TCP friendly streaming protocol is able to apply two kinds of protocol based congestion control: the window-based and the model-based. The window-based congestion control performs additive increase and multiplicative decrease (AIMD) rate adjustment which is similar to TCP.

As seen in Fig. 1, network estimation information is measured created (structured into metrics) and updated in Media Proxy/Gateway and End Nodes (mobile host) 
while it is transferred and continuously maintained at the application server. This scheme is required in order to provide the application server with all required information for create establish and manage an application session with an end node through a network of intermediate nodes. All former analysis has been presented here in order to define the environment that autonomous service management components will be specified as seen next.

\section{The Service Management Layer in GANA}

The next figure (fig 2) illustrates the structure of a GANA node its network and service layer DEs and how these DEs are managing its assigned MEs. For this study the DEs of prime interest are: the Service-Management-DE, Monitoring-DE, the Mobility-Management-DE, the QoS-Management-DE, the Forwarding-Management-DE and the Routing-Management-DE.

Each of these DEs, manages specific protocols and mechanisms assigned to it according to the aspects abstracted by the DE. The Routing-Management-DE manages the routing protocols and mechanisms of the node; the Forwarding-Management-DE manages the corresponding protocols for Layer-3/Layer-2.5/Layer Forwarding as well as Layer-3/Layer-2 Switching supported by the node (e.g. IPv6, MPLS, Ethernet). The Monitoring-DE manages those monitoring protocols and mechanisms that can be orchestrated for the benefit all the functions of the node including applications. The QoS-Management-DE manages the QoS related issues such as the schedulers and queue parameters on the interfaces and links connected to the node. The MobilityManagement-DE manages the mobility related mechanisms and protocols of the node.

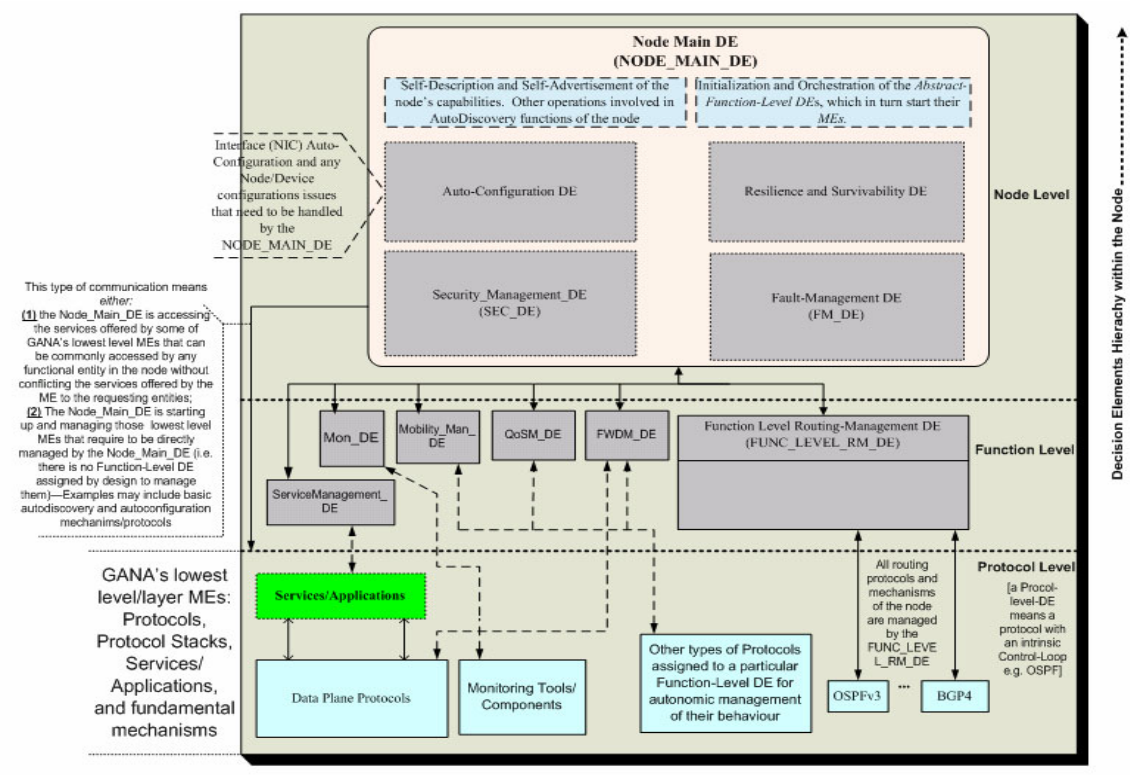

Fig. 2. The GANA Node with the Service Management Layer 
Finally the Node-Main-DE controls the overall behaviour of the node consisting of some sub-DEs (e.g. Security-Management-DE), which are considered part of the Node-Main-DE which require exclusive access to the lower DEs down to the lowest level of protocols and protocols stacks of the GANA node for the purposes of managing those aspects that are shared by all entities of the node or benefit them all.

\section{Autonomic Features under the Prism of Multimedia Delivery}

In order to efficiently make applications and services benefit from the autonomic features of the lower layers GANA introduces the need for interactions between network functions specific DEs and the Service-Management-DE in order to allow network functions specific DEs to know how to configure and manage their MEs, towards meeting the requirements imposed by Services and Applications, such as network resilience and QoS guarantees, etc. This creates the notion of a network-layer that is application and service-aware, including transport and network-layer awareness to survivability requirements of services and applications, which enables the network to react to the needs of applications and services. The survivability requirements of an application define the time constraints within which the application should be notified by the underlying transport and network layers of incidents requiring it to adapt to the challenging adverse conditions of the network.

In GANA, self-adaptation is implemented by the Forwarding-Management-DE or FWDM_DE (see the FWDM_DE on the Fig.2). All the DEs implement some kind of self-adaptation behaviors specific to the DE, which ensure that the node fulfils the optimal goal of its overall functionality and the performance of the applications and services running on the node. In [5] and [6] the reader can find an analysis of how self-adaptation on routing is achieved through the Routing-Management-DE and adaptive behaviors based the Monitoring-DE respectively. A representative paradigm of self-adaptation can be also found in [4] as implemented by the FWDM_DE for better understanding.

Based on the former analysis of the multimedia delivery aspects and involved functionality an interpretation of the described adaptation mechanisms into autonomous features in autonomic terms as follows:

- Media Codec Self-Adaptation: The autonomic node AND the autonomic server should be capable to self monitor the underlying application session conditions and connection environment, exchange information between them about network conditions estimation and decide about the most suitable coding mechanism that will guarantee the particular QoS level(s) at the end node and servicing network.

- Service/Application Self-Management: The autonomic node after getting continuous monitoring information about its state, the connected access network conditions and the application quality at the end user it decides about the necessary parameter change(s) to be requested from other autonomic components (e.g. QoS components) in order to protect or maintain the agreed application/service quality. 
- Service/Application Self-Healing: The autonomic node in connection with other autonomic DEs and the autonomic application server will decide about the policy/strategy to be applied in the case of application collapse due to specific reason(s) identified and known to the autonomic node (e.g. congestion, channel fading, packet loss).

\section{GANA-Based Service Management for Streaming Media}

\subsection{Requirements}

The Service Management autonomic component called Service Management Decision Element (SM_DE) has the following roles:

- Guarantee the required network resources (through QoS requests) the protocol-level session management entities like SIP/SDP, RTP/RTCP, RTSP, etc, considered as Managed Entities (MEs) in the GANA architectural Reference Model.

- Monitor the application level performance metrics through communication with the Service-related Managed Entities (e.g. streaming packets delay \& loss through RTCP)

- Guarantee the best possible service quality and experience at the end user interface

- Continuously balance and maintain service stability between applicationlevel quality and network resource availability

From the functional perspective the SM_DE is expected to manage all underlying lower protocol level entities under the general term Session-associated Managed Entities (MEs) responsible for service discovery, registration, initiation, control, etc, performing the following tasks:

- Request the service initiation (in the case of SIP, SDP) to launch the desired service after contacting the remote application server (e.g. INVITE, ACK)

- In the case of multimedia communication it activates and manages the $\mathrm{RTP} / \mathrm{RTCP}$ protocols in order to efficiently control the formerly launched session.

- After successful session launch, it continuously monitors its progress and performance by exchanging information about perceived service quality (streaming packet loss and delay) between end points.

- Contact QoS Management DE in the same layer (e.g. Function-Level in GANA) in order to negotiate service specific QoS levels

- Continuously receive monitoring information from the Monitoring DE about underlying network performance in order to maintain a combined view of network and transport conditions the active session runs with.

\subsection{Proposed Framework for Service Management}

The Service Management DE or SM_DE is the core component for GANA-based (fig. 3) for autonomic service management that is responsible for: 
- Manage the session and application level mechanisms at the lower protocol level (e.g. RTP, RTCP) in the case that running application(s) require more media resources (e.g. better video quality, higher data rate) by contacting the remote application server.

- Request more network resources (e.g. increase QoS, stronger channel coding due to errors in wireless transmission) in the case of bandwidth greedy application by contacting directly other DEs at the same layer (e.g. QoS_DE)

- Continuously monitor the network performance metrics (delay, loss, jitter) in close connection with Monitoring_DE and cross check the observed network conditions with the application level metrics (e.g. congestion, delay, loss) in order to act proactively about an upcoming application collapse event (it may consult application server through RTCP to reduce streaming packet rate due to the detection of bandwidth fluctuations at the end user)

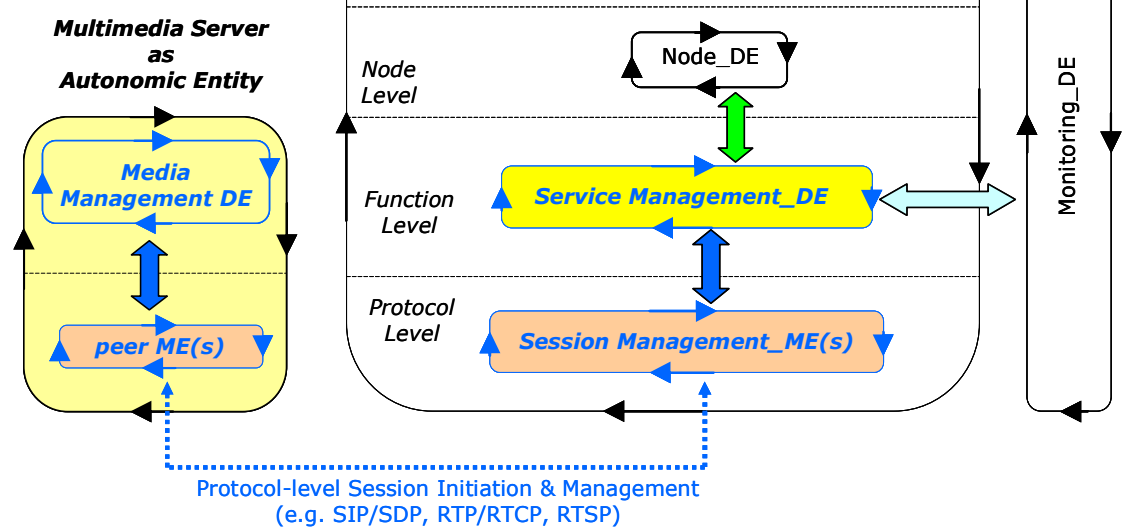

Fig. 3. Service Management ecosystem in GANA

At the protocol level the Session Management Managed Entity (Session Management $\mathrm{ME}$ ) in one of the known transport level protocols for service management like SIP/SDP, RTP/RTCP, etc as described in chapter 2. This ME is managed directly by the SM_DE above and do not take decisions about the session before it contact SM_DE. Obviously in this way the SM_DE is the coordinating entity that upon getting a clear view of existing conditions on the network and application parts decides about the required modifications in system parameters regarding performance and end user application quality. 


\section{Conclusions and Future Work}

In this paper the high-level requirements for multimedia delivery in an autonomic network exploiting the benefits of cross-layer techniques is presented. The main focus was on the emerged evolvable holistic architectural based on GANA Reference Model for autonomicity and self-management within node and network architectures and their proposed extension for services and application layer. In order to efficiently make applications and services benefit from the autonomic features of the GANA lower layers a number of interactions between network level Decision Elements (DEs) and the Service Management DE has been introduced in order to allow network function specific DEs to know how to configure and manage their Managed Entities (MEs), towards meeting the requirements imposed by Services and Applications, such as network resilience and QoS guarantees, etc. Furthermore self-adaptation of DEs itself is significantly improved ensuring that the node fulfils the optimal goal of its overall functionality while the performance of node's running services and applications as well.

\section{Acknowledgement}

This work has been partially supported by EC FP7 EFIPSANS project (INFSO-ICT215549).

\section{References}

1. EU funded FP7-EFIPSANS Project, http: / / efipsans . org/

2. Zhang, Q., Yang, F., Zhu, W.: Cross-Layer QoS Support for Multimedia Delivery over Wireless Internet. EURASIP Journal on Applied Signal Processing 2005(2), 207-215 (2005)

3. Chaparadza, R., Papavassiliou, S., Kastrinogiannis, T., Vigoureux, M., Dotaro, E., Davy, A., Quinn, K., Wódczak, M., Toth, A., Liakopoulos, A., Wilson, M.: Creating a viable Evolution Path towards Self-Managing Future Internet via a Standardizable Reference Model for Autonomic Network Engineering. In: FIA Prague 2009 Conference, Published in the Future Internet Book produced by FIA (2009)

4. Chaparadza, R., Prakash, A.: Self-configuring and Self-Adaptive Forwarding in the GANA based Self-Managing Future Internet Architecture. Submitted to the 2nd Workshop on Monitoring, Adaptation and Beyond (MONA+), November 23 or 24 (2009), co-organized with the ICSOC/ServiceWave 2009 Conference, Stockholm, Sweden

5. Rétvari, G., Németh, F., Chaparadza, R., Szabó, R.: OSPF for Implementing Self-adaptive Routing in Autonomic Networks: a Case Study. In: Proceedings of the the 4th IEEE International Workshop on Modelling Autonomic Communication Environments (MACE 2009), Venice, Italy, October 26-27 (2009)

6. Zafeiropoulos, A., Liakopoulos, A., Davy, A., Chaparadza, R.: Monitoring within an Autonomic Network: A GANA based Network Monitoring Framework. Submitted to the 2nd Workshop on Monitoring, Adaptation and Beyond (MONA+), November 23 or 24 (2009); co-organized with the ICSOC/ServiceWave 2009 Conference, Stockholm, Sweden

7. Cheng, Y., et al.: A generic architecture for autonomic service and network management. Computer Communications Journal, Copyright 2006 Elsevier B.V. All rights reserved (2006), doi:10.1016/j.comcom.2006.06.017 